\title{
Response of the Duffing-van der Pol oscillator under position feedback control with two time delays
}

\author{
Xinye $\mathrm{Li}^{\mathrm{a}, *}$, Huabiao Zhang ${ }^{\mathrm{b}}$ and Lijuan Zhang ${ }^{\mathrm{a}}$ \\ a School of Mechanical Engineering, Hebei University of Technology, Tianjin, China \\ ${ }^{\mathrm{b}}$ School of Astronautics, Harbin Institute of Technology, Harbin, China
}

Received 8 February 2010

Revised 25 April 2010

\begin{abstract}
In this paper, the dynamics of Duffing-van der Pol oscillators under linear-plus-nonlinear position feedback control with two time delays is studied analytically and numerically. By the averaging method, together with truncation of Taylor expansions for those terms with time delay, the slow-flow equations are obtained from which the trivial and nontrivial solutions can be found. It is shown that the trivial solution can be stabilized by appropriate gain and time delay in linear feedback although it loses its stability via Hopf bifurcation and results in periodic solution for uncontrolled systems. And the stability of the trivial solution is independent of nonlinear feedback. Different from the case of the trivial solution, the stability of nontrivial solutions is also associated with nonlinear feedback besides linear feedback. Non-trivial solutions may lose their stability via saddle-node or Hopf bifurcation and the resulting response of the system may be quasi-periodic or chaotic. The feedback gains and time delays have great effects on the amplitude of the periodic solutions and their bifurcation control. The simulations, obtained by numerically integrating the original system, are in good agreement with the analytical results.
\end{abstract}

\section{Introduction}

As the combination of the two typical nonlinear systems, Duffing-van der Pol oscillators have received considerable interest since they can be used as models in physics, engineering, electronics, biology, neurology and many other disciplines [1-7]. In the last decade, much effort has been devoted to controlling the complicated dynamic behavior of nonlinear systems by using a feedback control with time delay [8]. Hu et al. [9] considered the primary resonance and the 1:3 subharmonic resonance of a Duffing oscillator under linear state feedback control with a time delay. Maccari [10] developed an asymptotic perturbation method when investigating the parametric resonance of a van der Pol oscillator under state feedback control with a time delay. Zhu et al. [11] applied a new stochastic averaging method to predict the response of a Duffing-van der Pol oscillator under both external and parametric excitation of wide-band stationary random processes. Xu et al. [12] discussed the primary resonant dynamics of an externally excited van der Pol-Duffing oscillator under time-delayed position feedback control and found two routes to chaos, namely period-doubling bifurcation and torus breaking. Kakmeni et al. [13] examined the strange attractors and chaos control in an anharmonic Duffing-van der Pol oscillator with two external periodic forces by applying numerical results and presented a control scheme for chaos suppression. Li et al. [14] considered the response of a forced Duffing-van der Pol oscillator under weak linear-plus-nonlinear state feedback control with a time delay and found that unwanted multiple solutions can be eliminated. It is also shown that coupled nonlinear state feedback control can

*Corresponding author. E-mail: address:xinyeli1966@yahoo.com.cn. 
be replaced by uncoupled nonlinear state feedback control. In another paper, Li et al. [15] investigated the dynamical behavior of a parametrically excited Duffing-van der Pol oscillator under linear-plus-nonlinear state feedback control with a time delay. It was demonstrated that nontrivial steady state response may lose their stability by saddle-node or Hopf bifurcation when parameters such as feedback gains and time delays vary. Ji et al. drew attention to a forced Duffing-van der Pol oscillator under weak linear-plus-nonlinear state feedback control with a time delay. It was found that the trivial equilibrium of the autonomous system may lose its stability via subcritical or supercritical Hopf bifurcation and the forced periodic response may lose its stability via Neimark-Sacker bifurcation [16]. ELBassiouny [17] studied the fundamental resonance and subharmonic resonance of order one-half of a harmonically forced oscillation under state feedback control with a time delay by using the multiple scale perturbation technique. Li Jun et al. [18] investigated the high-amplitude response suppression of the primary resonance of a nonlinear plant under cubic velocity feedback by means of the multiple scales method. It was demonstrated that appropriate choice for the feedback gain can greatly reduce the response amplitude of the primary resonance and completely eliminate the multiple responses. Maccari [19] studied the periodic solutions for the fundamental resonance response of a van der Pol-Duffing system under time-delayed linear position and velocity feedback using the asymptotic perturbation method. It was demonstrated that stable periodic solutions with arbitrarily chosen amplitude can be accomplished if the vibration control terms are added. Ji et al. explored three types of additive resonance response [20], two types of difference resonance response [21] and two types of primary resonance at non-resonant bifurcations of co-dimension two [22] respectively. However, the dynamical behavior of the Duffing - van der Pol system under linear-plus-nonlinear position feedback control with two time delays, which is described by Eq. (1), has not been presented up to now.

$$
\ddot{x}+\omega_{0}^{2} x-\varepsilon\left(\alpha-\gamma x^{2}\right) \dot{x}+\varepsilon \beta x^{3}=\varepsilon\left[g_{1} x\left(t-\tau_{1}\right)+g_{2} x^{3}\left(t-\tau_{2}\right)\right]
$$

where the dot denotes differentiation with respect to physical time $t, \omega_{0}$ is the nature frequency, $\alpha$ and $\gamma$ are the linear and nonlinear damping coefficients with $\alpha>0, \beta$ is the coefficient of the nonlinear stiffness, $\varepsilon$ is a small positive parameter, $g_{i}$ and $\tau_{i}(i=1,2)$ are the feedback gains and time delays respectively.

The present work aims at investigating the effects of gains and time delays in feedback control on the response of the system considered. The remainder of this paper is arranged as follows. In Section 2, the slow- flow equations, of which the fixed points correspond to different types of solutions of the system considered are derived with the aid of the method of averaging. In Sections 3 and 4, the stability of the trivial and nontrivial solutions is discussed respectively along with simulations. The paper closes with a discussion and some conclusions in Section 5.

\section{Slow-flow equations}

Equation (1) can be rewritten as

$$
\ddot{x}+\omega_{0}^{2} x=\varepsilon F\left(x, \dot{x}, x\left(t-\tau_{1}\right), x\left(t-\tau_{2}\right)\right)
$$

where

$$
F=\left(\alpha-\gamma x^{2}\right) \dot{x}-\beta x^{3}+g_{1} x\left(t-\tau_{1}\right)+g_{2} x^{3}\left(t-\tau_{2}\right)
$$

For the case of small $\varepsilon$, the approximate solution of Eq. (2) is supposed to take the following form according to the method of averaging [23-25],

$$
x=R \cos \left(\omega_{0} t-\theta\right)
$$

where the amplitude $R$ and the phase $\theta$ are time-dependent and governed by

$$
\begin{aligned}
& \dot{R}=-\varepsilon \begin{array}{c}
1 \\
2 \pi
\end{array} \int_{0}^{2 \pi} \omega_{0}^{\omega_{0}} \sin \left(\omega_{0} t-\theta\right) F d t \\
& R \dot{\theta}=\varepsilon \begin{array}{c}
1 \\
2 \pi
\end{array} \int_{0}^{\omega_{0}^{2 \pi}} \cos \left(\omega_{0} t-\theta\right) F d t
\end{aligned}
$$




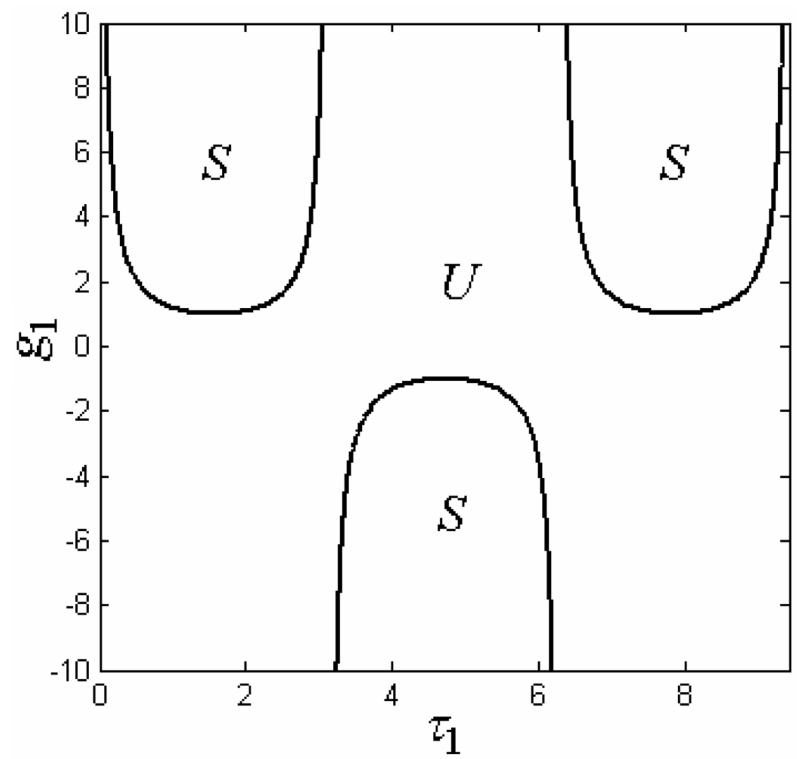

Fig. 1. The boundary where Hopf bifurcation of the trivial solution occurs in the $\tau_{1}-g_{1}$ plane, $\omega_{0}=\alpha=1$

After integrating and truncating the Taylor expansions for those terms containing time delay [26], Eq. (5) is in the following form, namely, slow-flow equations

$$
\begin{aligned}
& 8 \omega_{0} \dot{R}=-R\left[4 g_{1} \sin \left(\omega_{0} \tau_{1}\right)+R^{2} \gamma \omega_{0}+3 g_{2} R^{2} \sin \left(\omega_{0} \tau_{2}\right)-4 \alpha \omega_{0}\right] \\
& 8 \omega_{0} R \dot{\theta}=-R\left[3 \beta R^{2}-4 g_{1} \cos \left(\omega_{0} \tau_{1}\right)-3 g_{2} R^{2} \cos \left(\omega_{0} \tau_{2}\right)\right]
\end{aligned}
$$

where the dot indicates differentiation with respect to the rescaled time $T=\varepsilon t$. Equation (6) is an autonomous dynamical system, the nontrivial fixed points of which correspond to the periodic motions of the system described by Eq. (1). It is clearly seen that the addition of feedback control modifies the modulation equations. Consequently, with feedback control it is possible to change the nonlinear dynamic characteristics of the system.

If $\tau_{1}=\tau_{2}=0$ in Eq. (6), i.e., the time delays in feedback are zero, the slow flow equations for the system under feedback control without time delay are obtained. Similarly, the slow flow equations for the case without feedback control can be obtained by setting $g_{1}=g_{2}=0$ in Eq. (6). Equation (6) has both trivial and nontrivial fixed points, and the later corresponds to the periodic response of Eq. (1).

\section{Stability and bifurcation of the trivial solution}

It is necessary to express Eq. (6) in Cartesian form to avoid being divided by zero when considering the stability of the trivial solution. For this purpose, the approximate solution (4) can alternatively be written as

$$
x=p \cos \omega_{0} t+q \sin \omega_{0} t
$$

where $p$ and $q$ are determined by

$$
\begin{aligned}
8 \omega_{0} \dot{p} & =p\left[-4 g_{1} \sin \omega_{0} \tau_{1}+4 \alpha \omega_{0}-\left(p^{2}+q^{2}\right)\left(\omega_{0} \gamma+3 g_{2} \sin \omega_{0} \tau_{2}\right)\right] \\
& +q\left[-4 g_{1} \cos \omega_{0} \tau_{1}+\left(p^{2}+q^{2}\right)\left(3 \beta-3 g_{2} \cos \omega_{0} \tau_{2}\right)\right] \\
8 \omega_{0} \dot{q} & =p\left[4 g_{1} \cos \omega_{0} \tau_{1}-\left(p^{2}+q^{2}\right)\left(3 \beta-3 g_{2} \cos \omega_{0} \tau_{2}\right)\right] \\
& +q\left[-4 g_{1} \sin \omega_{0} \tau_{1}+4 \alpha \omega_{0}-\left(p^{2}+q^{2}\right)\left(\omega_{0} \gamma+3 g_{2} \sin \omega_{0} \tau_{2}\right)\right]
\end{aligned}
$$

The eigenvalues of the Jacobian matrix for the trivial solution are the roots of the following equation 


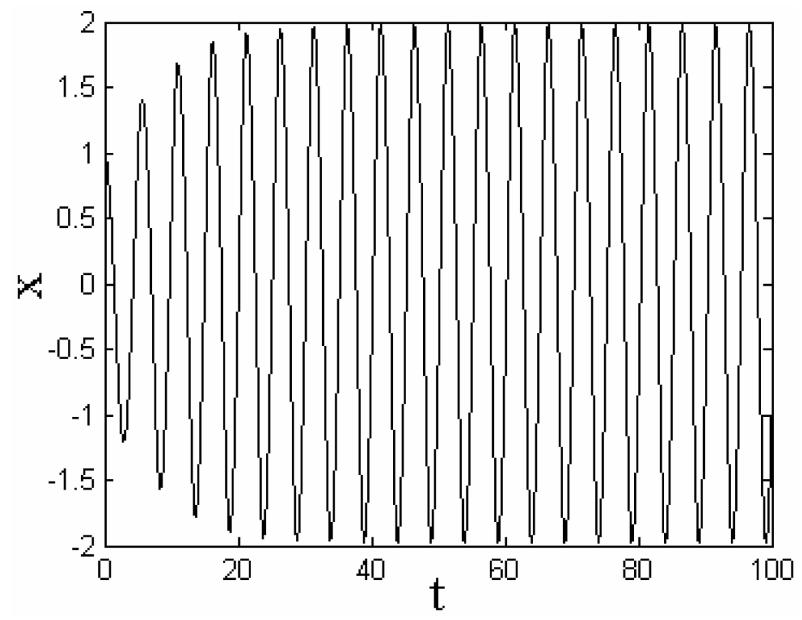

Fig. 2. Time history of the uncontrolled system, $\omega_{0}=\alpha=\beta=\gamma=1, \varepsilon=0.2$.

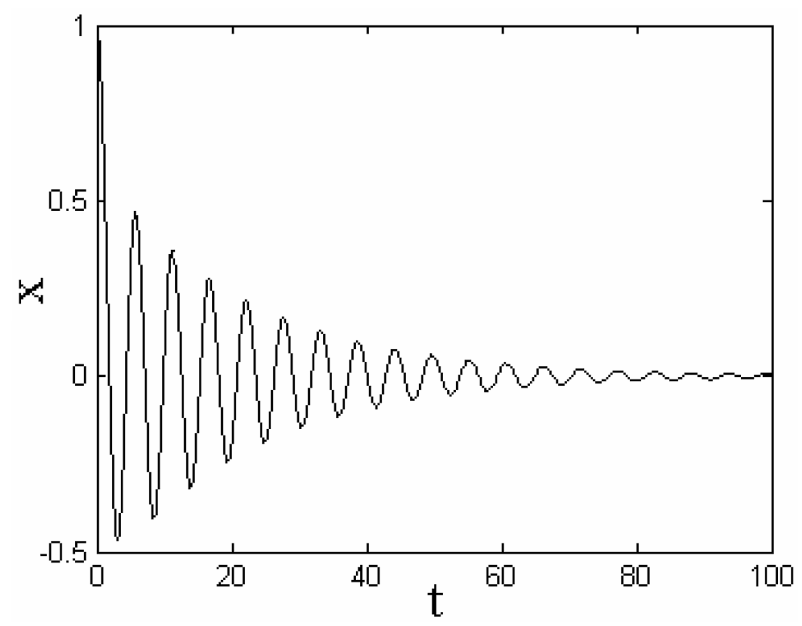

Fig. 3. Time history of the controlled system with parameters of linear feedback in"S"region of Fig. 1. $\omega_{0}=\alpha=\beta=\gamma=1, \varepsilon=0.2, g_{1}=2$, $\tau_{1}=2, g_{2}=0$.

$$
\lambda^{2}+2 a_{1} \lambda+a_{2}=0
$$

where $a_{1}=4 g_{1} \sin \omega_{0} \tau_{1}-4 \alpha \omega_{0}, a_{2}=\left(4 g_{1} \sin \omega_{0} \tau_{1}-4 \alpha \omega_{0}\right)^{2}+\left(4 g_{1} \cos \omega_{0} \tau_{1}\right)^{2}$. According to the Routh-Hurwitz criterion, the trivial solution is asymptotically stable if and only if $a_{1}>0$ and $a_{2}>0$ hold simultaneously. Therefore the boundary between the stable and unstable regions of the trivial solution is $a_{1}=0$ since $a_{2}>0$ is satisfied by definition. The condition necessary for Hopf bifurcation of the trivial solution is $a_{1}=0$ which means that a pair of purely imaginary eigenvalues exists. The curve determined by $a_{1}=0$ is as shown in Fig. 1 where $\mathrm{S}$ and $\mathrm{U}$ denote stable and unstable regions respectively.

$$
g_{1} \sin \omega_{0} \tau_{1}-\alpha \omega_{0}=0
$$

The trivial solution of the uncontrolled system is unstable since $a_{1}<0$, which is demonstrated in Fig. 2. By appropriate gain and time delay in linear feedback, it is possible to keep $a_{1}>0$. Therefore the trivial solution can be stabilized by feedback control as shown in Fig. 3, which means its Hopf bifurcation can be removed. Certainly, the trivial solution is still unstable if linear feedback parameters are not appropriate, which is shown in Fig. 4. 


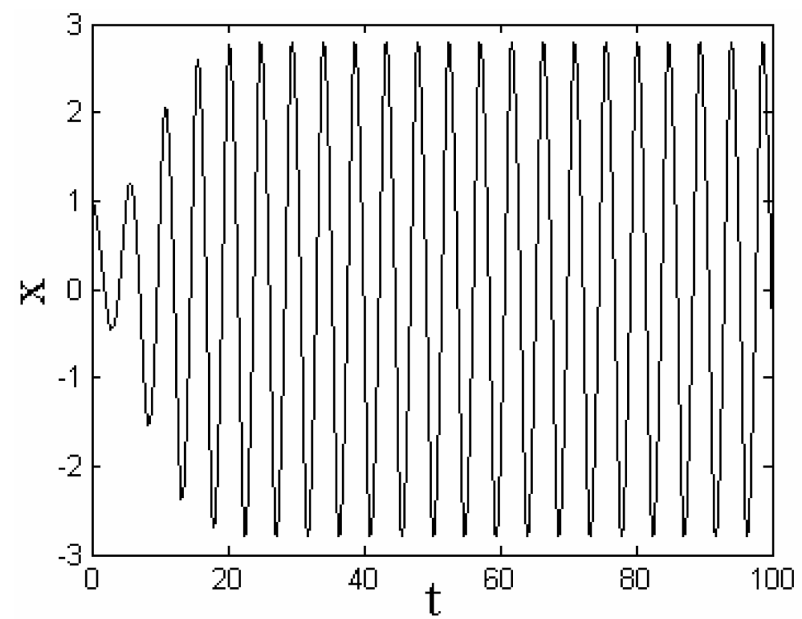

Fig. 4. Time history of the controlled system with parameters of linear feedback in "U" region of Fig. $1 . \omega_{0}=\alpha=\beta=\gamma=1, \varepsilon=0.2, g_{1}=$ $2, \tau_{1}=4, g_{2}=0$.

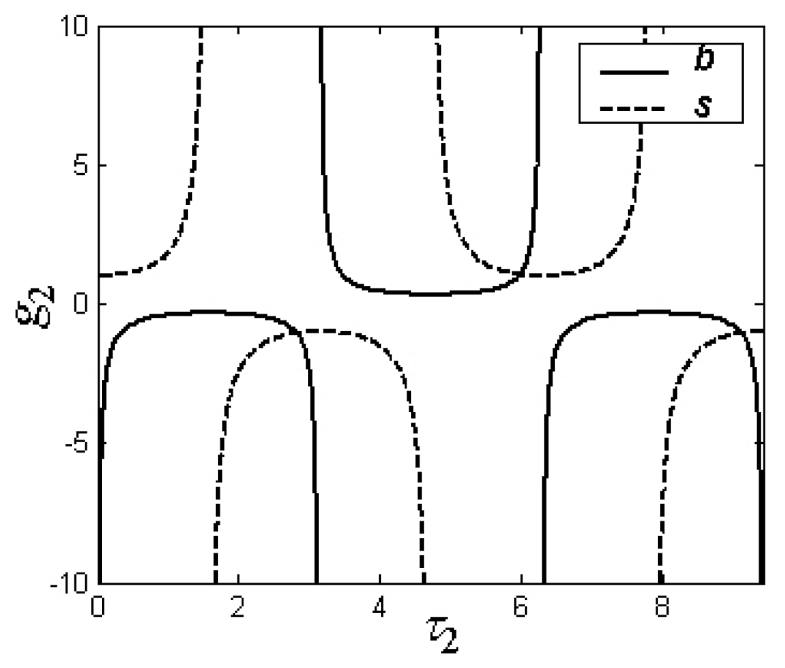

Fig. 5. The boundaries associated with the stability of periodic solutions in $\tau_{2}-g_{2}$ plane.

\section{Stability and bifurcation of periodic solutions}

In the previous section, it has been shown that when the parameters are varied to cross the critical boundary given by Eq. (10) due to time delay, the trivial solution loses its stability, leading to Hopf bifurcation (periodic) solutions. Letting $\dot{R}=0$ in Eq. (6), the amplitudes of the periodic solutions are determined by the following

$$
R^{2}=\begin{gathered}
4 \alpha \omega_{0}-4 g_{1} \sin \left(\omega_{0} \tau_{1}\right) \\
\gamma \omega_{0}+3 g_{2} \sin \left(\omega_{0} \tau_{2}\right)
\end{gathered}
$$

Case I) $\gamma \omega_{0}+3 g_{2} \sin \left(\omega_{0} \tau_{2}\right)=0$. This is the boundary between the two regions where periodic solutions exist and do not exist respectively. It is presented in the $g_{2}-\tau_{2}$ plane as shown in Fig. 5 (solid lines). For simplicity, values of parameters are chosen to be 1 in the following figures if not mentioned.

Case II) $\gamma \omega_{0}+3 g_{2} \sin \left(\omega_{0} \tau_{2}\right)>0$. Periodic solutions exist only when $4 \alpha \omega_{0}-4 g_{1} \sin \omega_{0} \tau_{1}>0$ holds. For $4 \alpha \omega_{0}-4 g_{1} \sin \omega_{0} \tau_{1}<0$, the trivial solution is stable, which has been shown in the last section. The condition $4 \alpha \omega_{0}-4 g_{1} \sin \omega_{0} \tau_{1}=0$ is just the boundary where the trivial solution loses its stability. Therefore, the periodic solution is the result of the bifurcation of the trivial solution. 

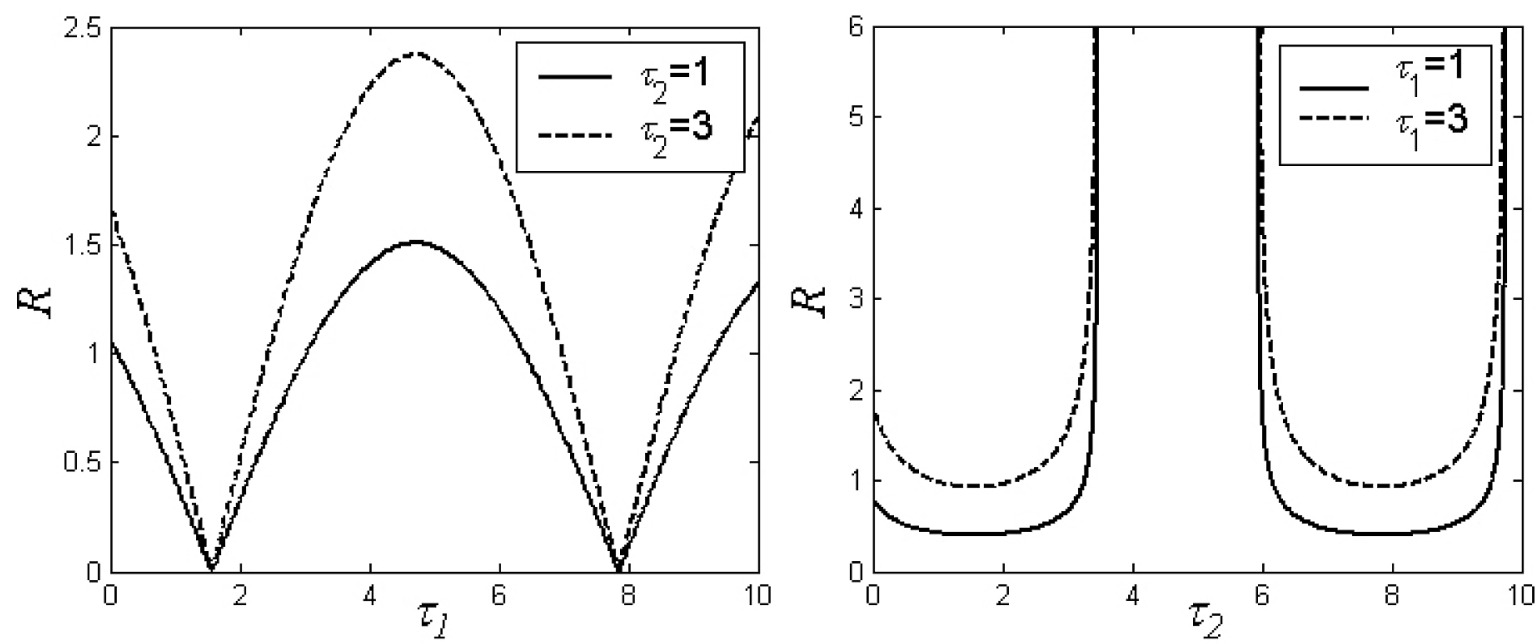

Fig. 6. The effect of time delays in feedback on amplitude of periodic motions.
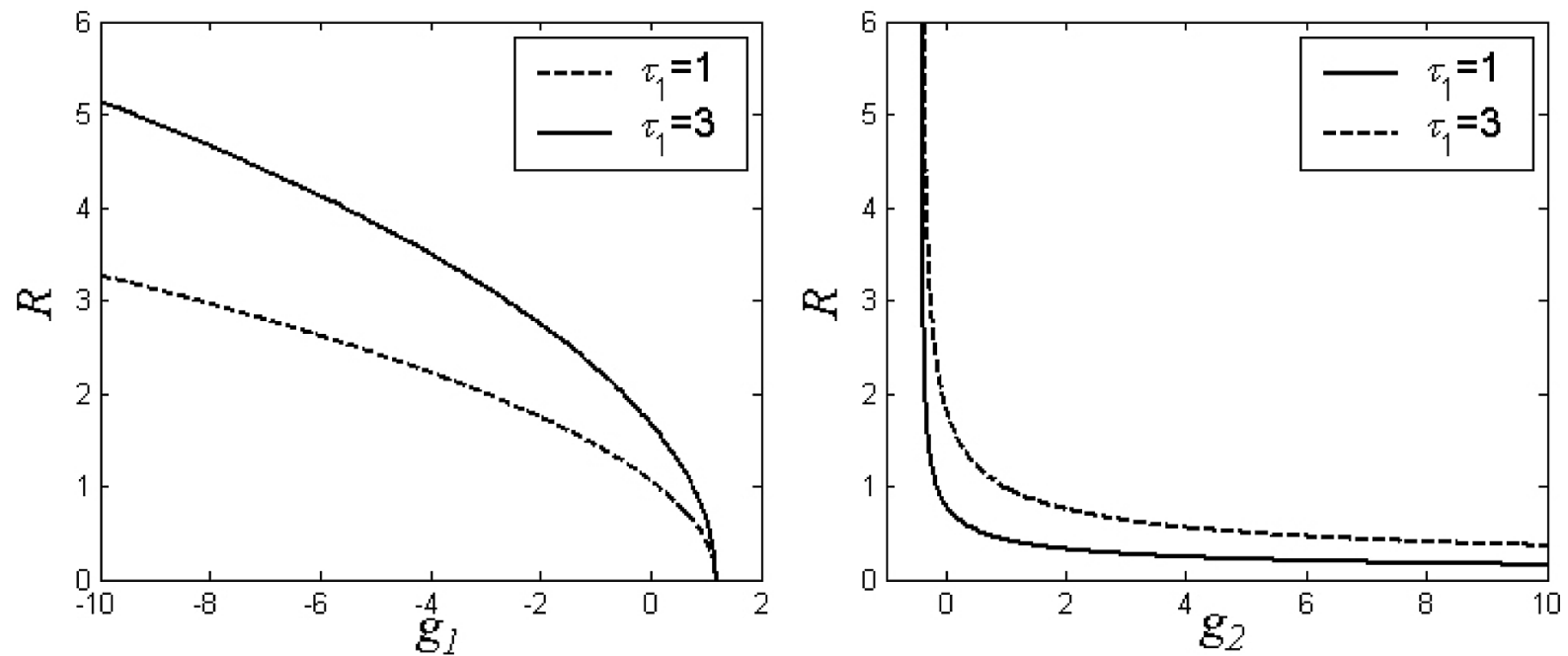

Fig. 7. The effect of feedback gains on amplitude of periodic motions.

Case III) $\gamma \omega_{0}+3 g_{2} \sin \left(\omega_{0} \tau_{2}\right)<0$. This is also possible if feedback gains are negative. Different from Case II), periodic solutions exist only when $4 \alpha \omega_{0}-4 g_{1} \sin \omega_{0} \tau_{1}<0$ holds. Therefore it is necessary to determine the stability of the periodic solutions since the trivial solution is stable when $4 \alpha \omega_{0}-4 g_{1} \sin \omega_{0} \tau_{1}<0$ holds. The eigenvalues of the Jacobian matrix for the periodic solutions determined by Eq. (11) are the roots of the following equation

$$
\lambda^{2}+2 b_{1} \lambda+b_{2}=0
$$

where $b_{1}=4 g_{1} \sin \omega_{0} \tau_{1}-4 \alpha \omega_{0}, b_{2}=6 R\left(\beta-g_{2} \cos \omega_{0} \tau_{2}\right)$. The conditions when Hopf bifurcation of periodic solutions occurs are given by $b_{1}=0$ when $b_{2}>0$ holds. It is obvious that $b_{1}$ is equal to $a_{1}$. Therefore the boundaries where Hopf bifurcation of the trivial solution occurs are the possible boundaries where Hopf bifurcation of the periodic solutions occurs. The condition necessary for saddle-node bifurcation of periodic solutions is given by $b_{2}=$ 0, i.e.,

$$
\beta-g_{2} \cos \omega_{0} \tau_{2}=0
$$




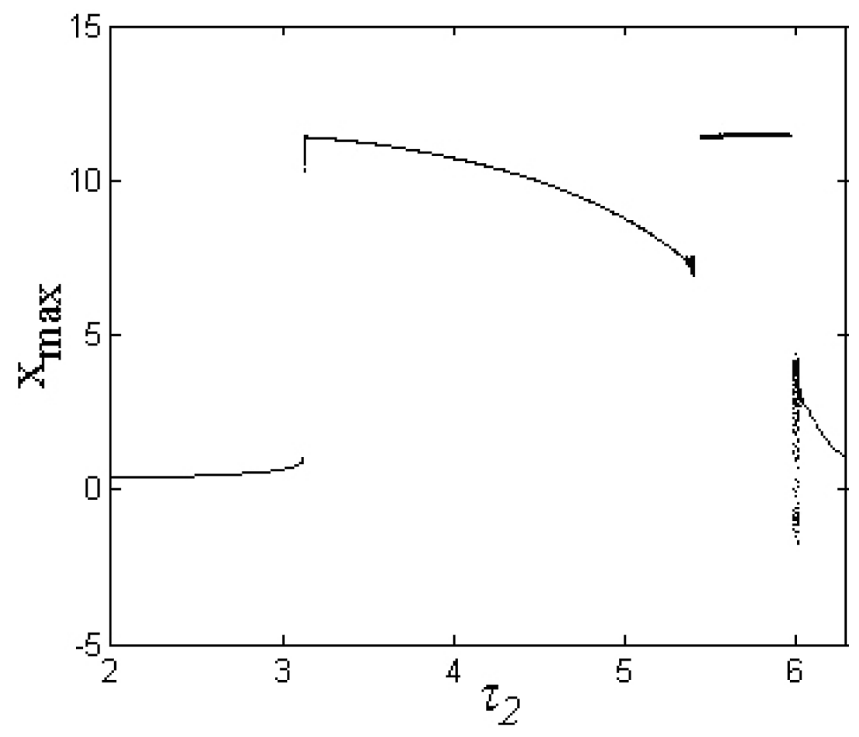

Fig. 8. Bifurcation diagram of response when time delay $\tau_{2}$ is varied.

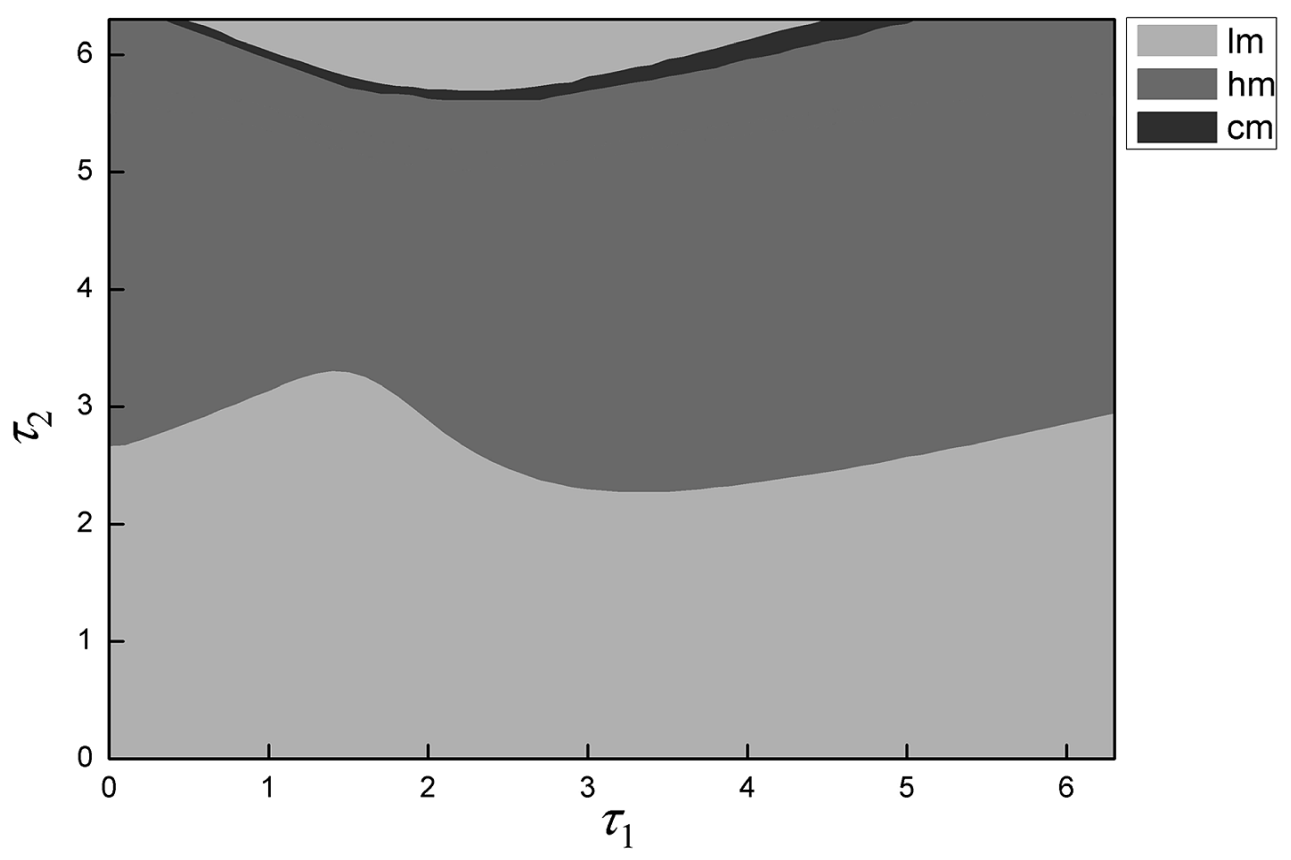

Fig. 9. Regions corresponding to different motions in the $\tau_{1}-\tau_{2}$ plane. (lm: low-amplitude motion, hm: high-amplitude motion, cm: complex motion).

This boundary is also presented in Fig. 5 where $\mathrm{b}$ and $\mathrm{s}$ denote boundaries determined by $\gamma \omega_{0}+3 g_{2} \sin \left(\omega_{0} \tau_{2}\right)=0$ and Eq. (13) respectively.

The effect of feedback on the amplitude of periodic solutions can be graphically presented as shown in Figs 6 through 7 from which those regions where periodic solutions do not exist can be found. In Fig. 8, the bifurcation diagram of response is directly obtained from Eq. (1) by numerical method, according to which it can be noted that the motion of the system can be periodic with lower amplitude or higher amplitude or complex motion when time delay $\tau_{2}$ is varied. In Fig. 9, the regions corresponding to different motions are given in the $\tau_{1}-\tau_{2}$ plane. To verify 

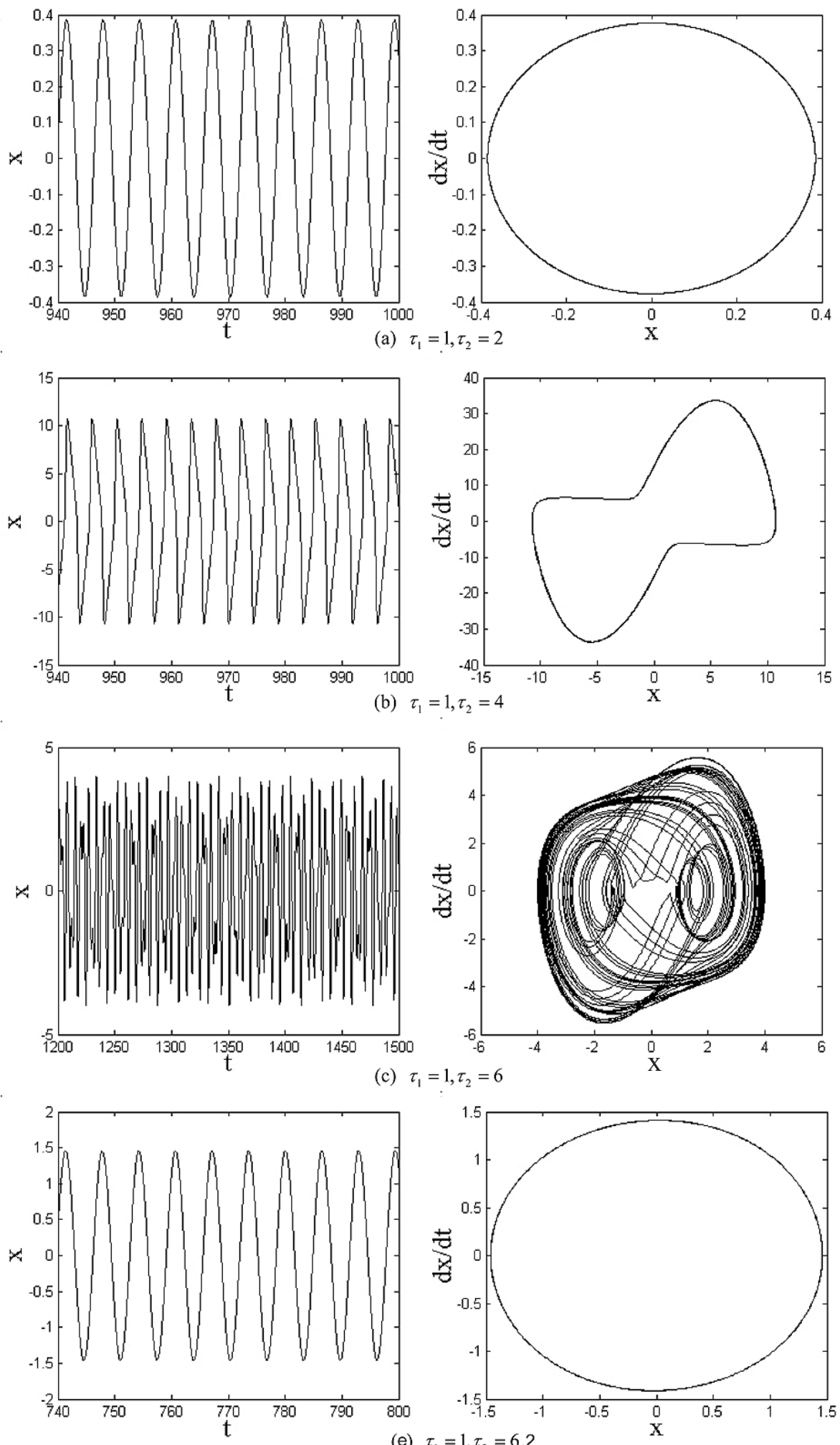

Fig. 10. Time history and trajectories corresponding to different time delays. 
the prediction in Fig. 9, some simulations, corresponding to different time delays, are given in Fig. 10 from which it is easy to note the effect of time delays in feedback on nontrivial solutions.

\section{Discussion and conclusions}

The dynamics of Duffing-van der Pol oscillators under linear-plus-nonlinear position feedback control with two time delays is investigated analytically and numerically in this paper. By the slow-flow equations, the stability of the trivial and nontrivial (periodic) solutions is discussed respectively. It is shown that the trivial solution can be stabilized by appropriate gain and time delay in linear feedback although it loses its stability via Hopf bifurcation and results in periodic solution if feedback control is not applied. Different from the case of the trivial solution, the stability of periodic solutions is also associated with the nonlinear feedback besides linear feedback. The feedback gains and time delays have great effects on the amplitude of the periodic solutions. Periodic solutions may lose their stability via saddle-node or Hopf bifurcation and the resulting response of the system may be quasi-periodic or chaotic. The condition necessary for saddle-node bifurcation of periodic solutions is only depend on nonlinear feedback control, while the condition necessary for their Hopf bifurcation is only depend on linear feedback control. The simulations, obtained by numerically integrating the original system, are in good agreement with the analytical results.

\section{Acknowledgements}

This research is supported by the National Natural Science Foundation of China under Grant No.10872063. The authors would like to thank the reviewers for their valuable suggestions.

\section{References}

[1] J. Guckenheimer and P. Holmes, Nonlinear Oscillations, Dynamical Systems, and Bifurcations of Vector Fields, Springer, New York, 1983.

[2] J. Guckenheimer and P. Holmes, Nonlinear Oscillation and Bifurcation of Vector Field, Springer-Verlag, New York, 1993.

[3] G.V. Belyakova and L.A. Belyakov, On bifurcations of periodic orbits in the van der Pol-Duffing equation, International Journal of Bifurcation and Chaos 7 (1997), 459-462.

[4] W. Szemplinska Stupnicka and J. Rudowski, The coexistence of periodic, almost-periodic and chaotic attractors in the van der Pol-Duffing oscillator, Journal of Sound and Vibration 199 (1997), 165-175.

[5] A. Algaba, F. Fernandez-Sanchez, E. Freire, E. Gamero and A.J. Rodriguez-Luis, Oscillation-sliding in a modified van der Pol-Duffing electronic oscillator, Journal of Sound and Vibration 249 (2002), 899_-907.

[6] X.B. Liu and K.M. Liew, On the stability properties of a van der Pol-Duffing oscillator that is driven by a real noise, Journal of Sound and Vibration 285 (2005), 27-49.

[7] J.C. Ji, Nonresonant Hopf bifurcations of a controlled van der Pol-Duffing oscillator, Journal of Sound and Vibration 297 (2006), $183-199$.

[8] Y. Kuang, Delay differential equations with application in population dynamics, Springer, New York, 2002.

[9] H. Hu, E.H. Dowell and L.N. Virgin, Resonances of a harmonically forced Duffing oscillator with time delay state feedback, Nonlinear Dynamics 15 (1998), 311-327.

[10] A. Maccari, The response of a parametrically excited van der Pol oscillator to a time delay state feedback, Nonlinear Dynamics 26 (2001), $105-119$.

[11] W.Q. Zhu, Z.L. Huang and Y. Suzuki, Response and stability of strongly nonlinear oscillators under wide-band random excitation, International Journal of Nonlinear Mechanics 36 (2001), 1235-1250.

[12] J. Xu and K.W. Chung, Effects of time delayed position feedback on a van der Pol-Duffing oscillator, Physica D 180 (2003), $17-39$.

[13] F.M. Moukam Kakmeni, S. Bowong, C. Tchawoua and E. Kaptouom, Strange attractors and chaos control in a Duffing-Van der Pol oscillator with two external periodic forces, Journal of Sound and Vibration 277 (2004), 783-799.

[14] X. Li, J.C. Ji, C.H. Hansen and C.X. Tan, The response of a Duffing-van der Pol oscillator under delayed feedback control, Journal of Sound and Vibration 291 (2006), 644-655.

[15] X.-Y. Li, Y.-S. Chen, Z.-Q. Wu and T. Song, Response of parametrically excited Duffing-van der Pol oscillator with delayed feedback, Applied Mathematics and Mechanics 27 (2006), 1585-1595.

[16] J.C. Ji and C.H. Hansen, Stability and dynamics of a controlled van der Pol-Duffing oscillator, Chaos, Solitons and Fractals 28 (2006), $555-570$.

[17] A.F. EL-Bassiouny, Fundamental and subharmonic resonances of harmonically oscillation with time delay state Feedback, Shock and Vibration 13 (2006), 65-83. 
[18] L. Jun, Shen Rongying and Hua Hongxing, Cubic velocity feedback control of high-amplitude vibration of a nonlinear plant to a primary resonance excitation, Shock and Vibration 14 (2007), 1-14.

[19] A. Maccari, Vibration amplitude control for a van der Pol-Duffing oscillator with time delay, Journal of Sound and Vibration 317 (2008), 20-29.

[20] J.C. Ji and N. Zhang, Additive resonances of a controlled van der Pol-Duffing oscillator, Journal of Sound and Vibration 315 (2008), 22-33.

[21] J.C. Ji, N. Zhang and W. Gao, Difference resonances in a controlled van der Pol-Duffing oscillator involving time delay, Chaos, Solitons and Fractals 42 (2009), 975-980

[22] J.C. Ji and N. Zhang, Nonlinear response of a forced van der Pol-Duffing oscillator at non-resonant bifurcations of codimension two, Chaos, Solitons and Fractals 41 (2009), 1467-1475.

[23] A.H. Nayfeh and D.T. Mook, Non-linear Oscillations, Wiley, New York, 1979.

[24] A.H. Nayfeh, Perturbation Techniques, Wiley, New York, 1981.

[25] A.H. Nayfeh and B. Balachandran, Applied Nonlinear Dynamics, Wiley, New York, 1995.

[26] S. Wirkus and R. Rand, The dynamics of two van der Pol oscillators with delay coupling, Nonlinear Dynamics 30 (2002), 205-221. 

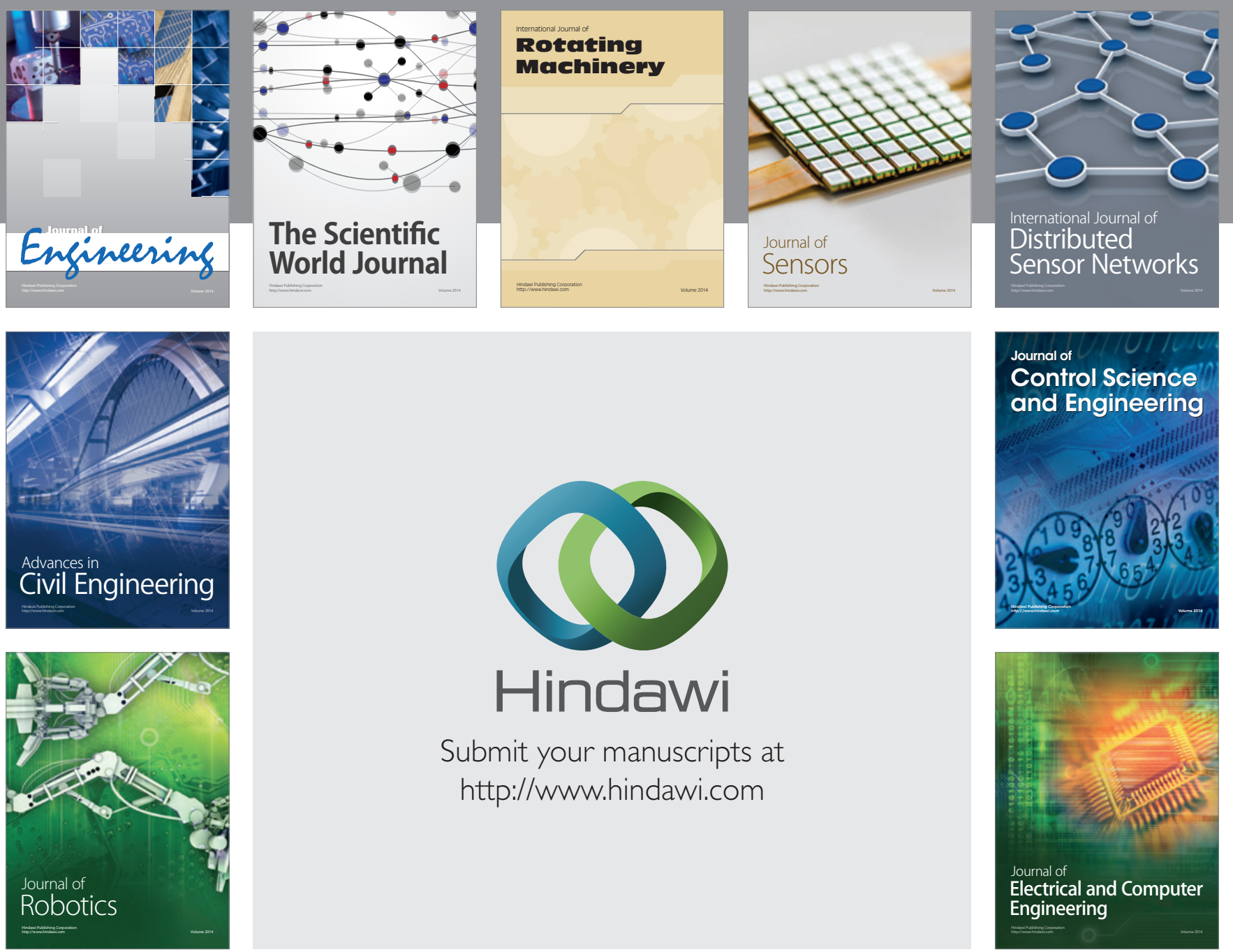

Submit your manuscripts at

http://www.hindawi.com
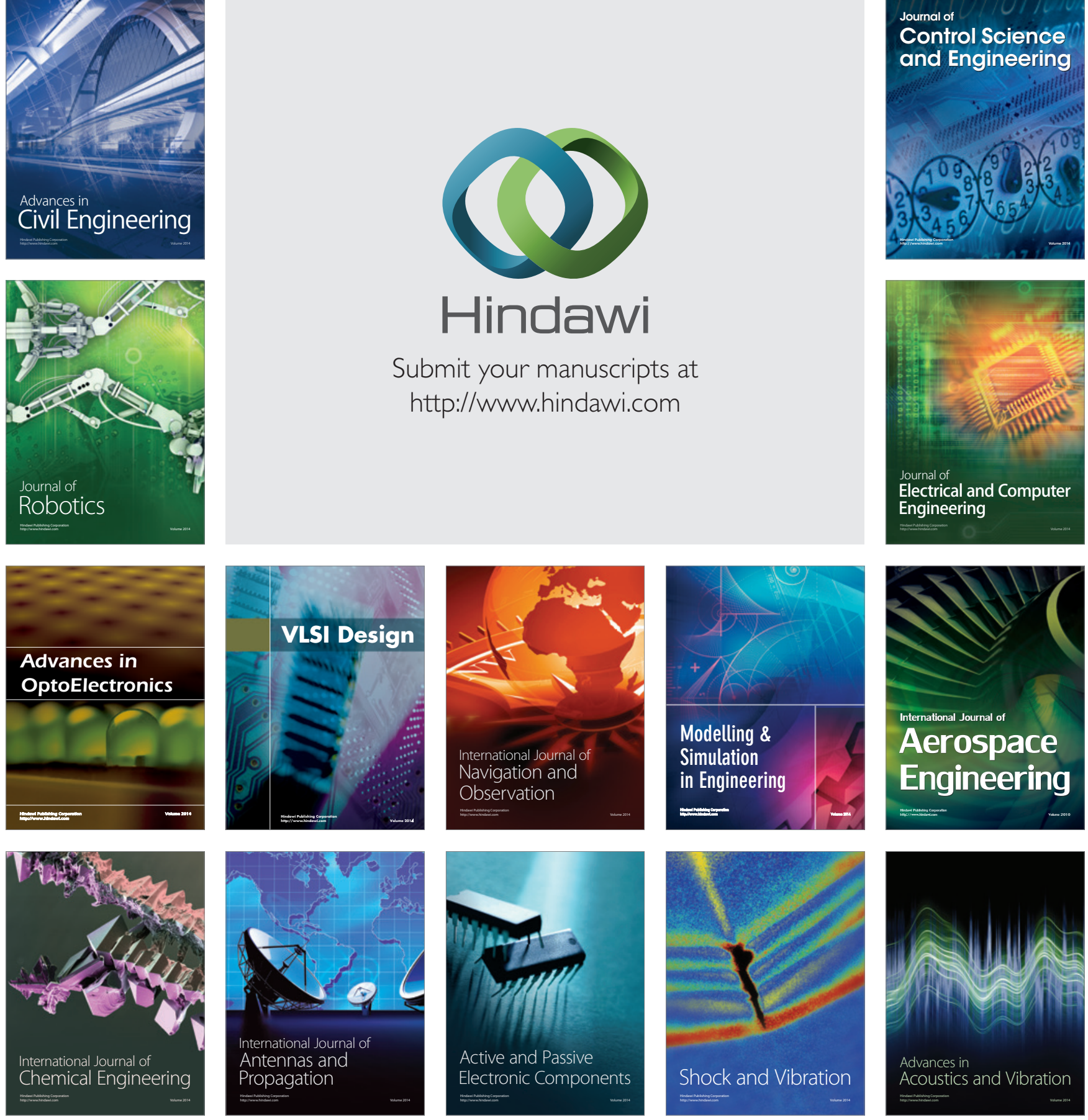